\section{Child and Adolescent Psychiatry: A Developmental Approach, 4th ed.}

by Jeremy Turk, Philip Graham, and Frank C. Verhulst. Oxford University Press, New York, New York, 2007, 579 pages, \$199.50, $\$ 70.00$ (paper).

The Fourth Edition of Child and Adolescent Psychiatry: A Developmental Approach, under the lead authorship of Jeremy Turk, proposes to offer a comprehensive resource suitable to the educational or clinical backgrounds of a full range of caring professionals attending to the psychosocial and psychiatric needs of children and adolescents. The volume is intended to complement standard pediatric texts, offering pragmatic, yet evidence-based and state-of-the-art clinical content addressing normal development and common problems through rare conditions and severe psychopathology while highlighting the unique contributions of each professional discipline to assessment and intervention. This singular text effectively reaches its interdisciplinary audience and clinical content objectives, as promised.

The organizational theme of the text is not dissimilar to the tiered model of health services for disturbed children and their families outlined in chapter 10 , which delineates services at the levels of primary care, professionals working as individuals, specialist multidisciplinary teams, and tertiary level services. The book is presented in 10 chapters: (1) "Introduction," (2) "Familial Influences," (3) "Neurodevelopment and Neuropsychiatric Disorders," (4) "Developmental Psychopathology," (5) "Adolescence and Psychiatric Disorders Often Beginning in Adolescence," (6) "Child-Adult Continuities in Psychiatric Disorders," (7) "Psychosocial Aspects of Physical Disorders," (8) "Psychological Aspects of Specific Physical Conditions," (9) "Prevention and Treatment," and (10) "Services." The subtopics in each chapter are organized numerically, with an organizational progression from general pediatric and developmental overviews to increasingly focused discussions of epidemiology, etiology, assessments, interventions, and outcomes. Discussions progress through each service tier of context, evaluation, and treatment.

The text's composition is polished and succinct without sacrificing technical salience. The authors include a number of wellchosen tables, practical diagrams, and case examples. The volume's greatest strength lies, however, in its conversational, yet scholarly style of writing in a distinctly pediatric voice. The authors convey an affecting sensitivity to the needs and care of parents, as well as a developmentally empathic approach to even the most troubled delinquent youth, evidence of the book's importance in forming students and trainees.

Nonpediatric readers will appreciate the inclusion of adjustment issues and specific treatment protocols for psychosocial conditions often treated in the pediatric setting, such as a primary care pathway in the management of attention-deficit/hyperactivity disorder (ADHD). Conversely, the introduction to operant change and outline of progressively advanced interventions in behavioral modification are another highlight of potential interest to pediatricians in general practice, who customarily refer serious behavior problems to specialty care. Although the chapter addressing services is largely specific to the United Kingdom, readers everywhere will appreciate the sections addressing prevention, special education, and professional treatment roles. If the text were to be found wanting at all, it might have been in my wish for a more expanded and detailed discussion of pediatric psychopharmacology.

This is a thoughtful and eloquently written text, which would serve equally well as a complement to either core pediatric or child psychiatry volumes. Novice and experienced physicians alike will appreciate the authors' fresh approach to the synthesis of interdisciplinary perspectives in a quality offering that succeeds in remaining comprehensive, practical, and relevant to daily practice. Educators in psychiatry, pediatrics, nursing, psychology, social work, and special education will view the Fourth Edition of Child and Adolescent Psychiatry: A Developmental Approach as an important resource in the training of clinically accomplished, empathic, and collaborative professionals prepared to participate in the care of childhood emotional difficulties and disorders.

Mary W. Roberts, MD mroberts@med.wayne.edu

Author affiliation: Wayne State University School of Medicine, Detroit, Michigan. Potential conflicts of interest: None reported. doi:10.4088/JCP.09bk05578whi

(c) Copyright 2010 Physicians Postgraduate Press, Inc 